\title{
BMJ Open Intradermal thumbtack needle buried Neiguan (P6) point for prevention of postoperative nausea and vomiting in patients undergoing craniotomy: study protocol for a randomised controlled trial
}

Jian-qin Lv, ${ }^{1}$ Chengwei Wang (D) , ${ }^{1}$ Yi Yang, ${ }^{2} \mathrm{Yu} \mathrm{Li},{ }^{2}$ Tian-hao Xu, ${ }^{2}$ Ling-qi $\mathrm{Jian}^{2}$

To cite: Lv J, Wang C, Yang Y, et al. Intradermal thumbtack needle buried Neiguan (P6) point for prevention of postoperative nausea and vomiting in patients undergoing craniotomy: study protocol for a randomised controlled trial. BMJ Open 2019;9:e032417. doi:10.1136/ bmjopen-2019-032417

- Prepublication history for this paper is available online. To view these files, please visit the journal online (http://dx.doi. org/10.1136/bmjopen-2019032417).

Received 21 June 2019 Revised 19 October 2019 Accepted 25 October 2019

A Check for updates

(C) Author(s) (or their employer(s)) 2019. Re-use permitted under CC BY-NC. No commercial re-use. See rights and permissions. Published by BMJ.

${ }^{1}$ Integrated Traditional and Western Medicine Department, Sichuan University West China Hospital, Chengdu, China

${ }^{2}$ West China School of Medicine, Sichuan University, Chengdu, China

Correspondence to Dr Chengwei Wang; wangchengwei@wchscu.cn

\section{ABSTRACT}

Introduction Postoperative nausea and vomiting (PONV) is among the most common adverse reactions following anaesthesia and surgery. Recent clinical studies have reported that the average incidence is about $30 \%$, while in patients specifically undergoing neurosurgery, the incidence can be as great as $73 \%$. Studies also suggest that its occurrence increases the risk of intracranial haematoma and haemorrhage. The objective of this study is to evaluate the effectiveness of intradermal thumbtack needle buried Neiguan (pericardium 6 (P6)) point therapy in the prevention of PONV in patients undergoing craniotomy under general anaesthesia.

Methods and analysis This is a single-centre, threearm, randomised controlled trial. 180 participants are randomly assigned to either an acupuncture, intradermal thumbtack needle or control group in a 1:1:1 ratio. The $\mathrm{P} 6$ of the acupuncture group is punctured at both sides perpendicularly to a depth of $20 \mathrm{~mm}$. Needles are retained for $30 \mathrm{~min}$ and stimulated every $10 \mathrm{~min}$ to maintain the de qi. The therapy includes two treatments; the acupuncture is administered immediately after and 24 hours after surgery. For the intradermal thumbtack needle group, the intradermal thumbtack needle is quickly inserted into the skin and embedded at P6 acupoints bilaterally. Patients and their families are asked to press the needlepoint with the onset of nausea, vomiting, bloating, pain and other reported discomforts. The needle is replaced after 24 hours. The therapy is administered immediately after and 24 hours after surgery. For the control group, no intervention is carried out. The incidence of PONV within 48 hours after craniotomy across the three groups is observed. Other observations include: (1) assessment of nausea score (severity of nausea) and pain score (visual analogue scale) 0-2, 2-6, 6-24 and 24-48 hours after craniotomy under general anaesthesia; (2) assessment of total rescue antiemetic dosage $0-48$ hours after craniotomy under general anaesthesia; (3) length of hospital stay and (4) patient satisfaction score with PONV management. We will perform all statistical analysis following the intention-to-treat principle.
Strengths and limitations of this study

- A randomised controlled trial of 180 patients will be conducted to evaluate the effectiveness of intradermal thumbtack needle buried Neiguan (pericardium 6) point therapy in the prevention of postoperative nausea and vomiting in patients undergoing craniotomy under general anaesthesia.

- The feasibility of the trial has been examined by a pilot randomised trial of 30 patients.

- This trial will be conducted using rigorous methods, such as the blinding of data analyst and outcome assessors, and the implementation of interventions using clearly prespecified approaches.

- The control group lacked placebo effect.

Ethics and dissemination Ethics approval has been granted by the Bioethics Subcommittee of the West China Hospital, Sichuan University: the approval number is 2018 (number 231). Results will be expected to be published in peer-reviewed journals.

Trial registration number ChiCTR1800017173

\section{BACKGROUND}

Postoperative nausea and vomiting (PONV) is one of the most commonly observed adverse reactions following anaesthesia and surgery. ${ }^{1-3}$ It increases the difficulty of medical care, delays the patient's recovery from anaesthesia, extends their hospital stay and increases overall healthcare costs. ${ }^{45}$ Recently, clinical studies report that there is a high incidence, about $30 \%$, of patients experiencing PONV. This is much higher in patients who specifically undergo neurosurgery. The incidence in these cases can be as high as $73 \%$. The studies also suggest that the occurrence of PONV can result in an increased risk of intracranial haematoma and haemorrhage. ${ }^{67}$ 
Pharmacological prophylaxis is widely used in clinical practice, and the most commonly used prophylactic antiemetics include serotonin (5-HT3) receptor antagonists, often in combination with either droperidol or dexamethasone. ${ }^{8-10}$ Recently, however, the US Food and Drug Administration has reported that droperidol may cause death secondary to arrhythmia or QT prolongation, while other drugs have varying degrees of side effects. ${ }^{11}$ At present, no therapy is categorically effective at preventing PONV.

Due to the limited efficacy and many side effects of drug therapy, various non-pharmacological techniques have been used in clinical practice. These therapies include acupuncture ${ }^{12}$ acupressure, ${ }^{13}$ transcutaneous electrical nerve stimulation (TENS), ${ }^{1415}$ and electroacupuncture, ${ }^{16}$ among others. ${ }^{17}$ In 2006, the American Society of PeriAnesthesia Nurses recommended pericardium 6 (P6; also known as Neiguan) acupoint stimulation (class IIb, grade A) and self-P6 acupoint compression before and after surgery (grade $\mathrm{C}$ ) as a complementary intervention for PONV prophylaxis. The 2014 American Anesthesia Outpatient Guide also recommended that acupuncture treatment may be used as an alternative or adjuvant therapy for the prevention of PONV. ${ }^{18}$ Many recent studies have supported the efficacy of $\mathrm{P} 6$ acupoint stimulation in preventing PONV. ${ }^{17}$ 19-21

The existing acupuncture treatment is still mainly focused on electroacupuncture or traditional needle operation. In our clinical practice, however, the limitations of these two acupuncture stimulation methods (including fixed treatment and fixed treatment time, inconvenience of other medical operations during needle retention, etc) have resulted in unstable efficacy.

A combination of traditional Chinese and Western medicine, including needle-embedding therapy, was put forward in the 1950s. With the developments of acupuncture apparatus, intradermal thumbtack needle has emerged as a new kind of embedding therapy. The intradermal thumbtack needle (figure 1), an improved subcutaneous needle, is a type of shallow needling method that reduces pain and prolongs the acupuncture effect via longer needle retention. Also, our previous clinical experience indicates that the time of nausea and vomiting in patients after surgery is uncertain. ${ }^{22-24}$ As the intradermal thumbtack needle has a 24 hours persistent stimulating effect, patients can self-press it at the P6 to relieve gastrointestinal discomfort when needed.

Therefore, we designed a single-centre, prospective, single-blind, randomised controlled trial (RCT) to evaluate the efficacy of $\mathrm{P} 6$ acupoint stimulation by intradermal thumbtack needle as a non-pharmacological prophylaxis for PONV. This study incorporates the concept of fasttrack surgery, using a prospective randomised controlled method, inspired by the concept of a postoperative analgesia pump, to develop P6 acupoint stimulation treatment by intradermal thumbtack needle with long-term stimulation characteristics (figure 2). The study is designed to evaluate the effectiveness and safety of P6 acupoint

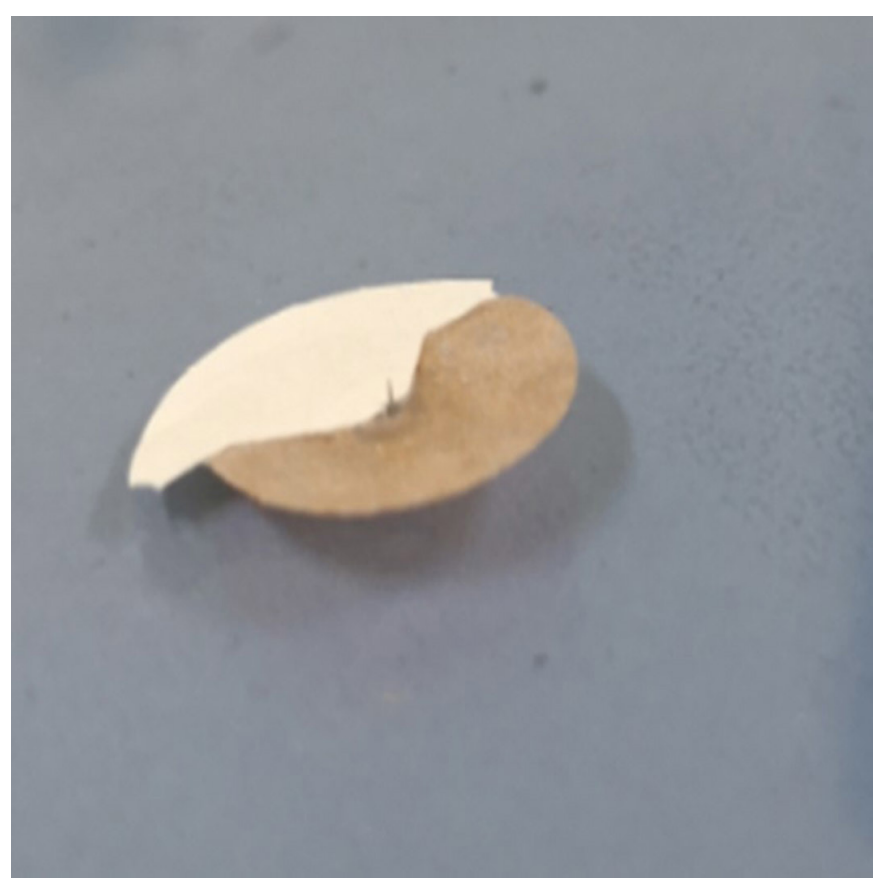

Figure 1 Intradermal thumbtack needle.

stimulation for the prevention of PONV in patients who undergo craniotomy under general anaesthesia by intradermal thumbtack needle versus acupuncture filiform needles, and versus routine antiemetic. The objective is to compare the effect and safety using different methods.

\section{METHODS \\ Design}

This is a single-centre, prospective, single-blind, parallelgroup, RCT. Participants have been and will continue to be recruited from the West China Hospital of Sichuan University (WCHSU) from January 2018 to November 2019. All participants are required to give written informed consent. The study's flow chart is shown in figure 3 .

\section{Patient population and setting}

A total of 180 Chinese patients undergoing craniotomy will be sequentially recruited at the WCHSU after fulfilling the eligibility criteria and signing the informed consent. A clinical assistant with institutional review board training will be in charge of patient enrolment.

\section{Eligibility criteria}

Inclusion criteria: Patients who fulfil the following conditions will be included: (1) scheduled for neurosurgery requiring opening of the cranium and dura; (2) aged between 18 and 70 years; (3) American Society of Anesthesiologists physical status classification of I or II; (4) undergoing general anaesthesia; (5) no history of PONV or motion sickness; (6) no experience with acupuncture therapies; (7) no use of antiemetic 24 hours before surgery; (8) willingness to participate and (9) having signed an informed consent form. 


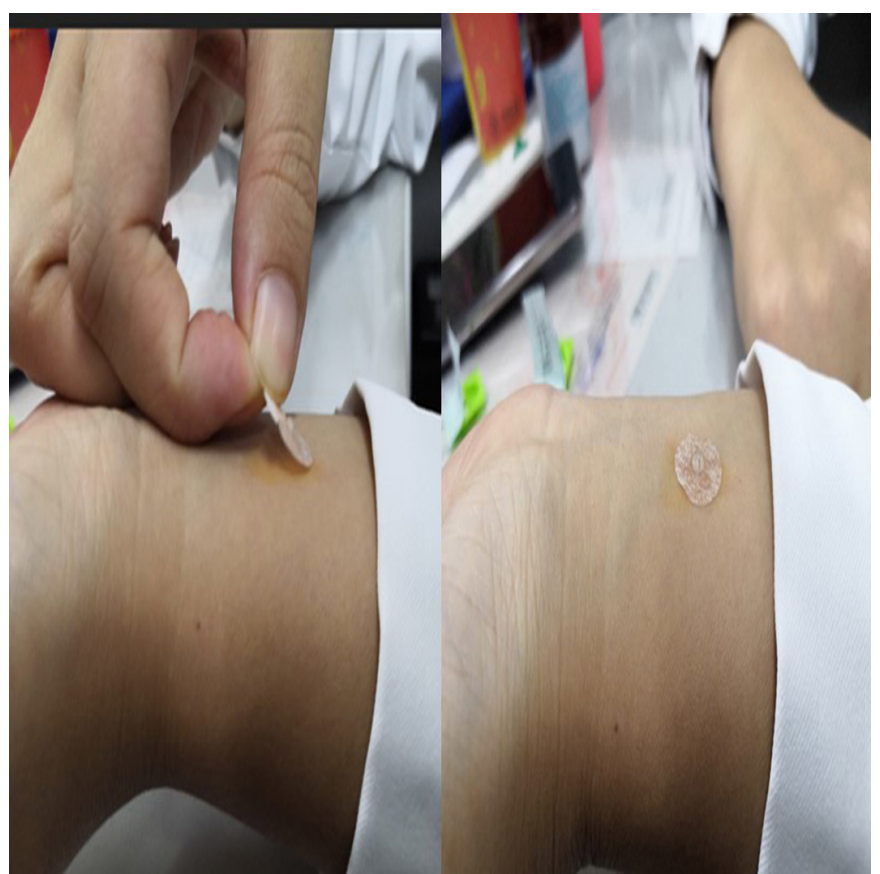

Figure 2 Intradermal thumbtack needle being inserted into the Neiguan point (P6). P6, pericardium 6.

Exclusion criteria: Participants that meet any of the following criteria will be excluded: (1) nausea or vomiting 24 hours before surgery; (2) pregnant or lactating women; (3) menstruating phase of the menstrual cycle; (4) drug or alcohol abusers; (5) criminals; (6) recipients of chemotherapy or radiation therapy during the previous 7 days before surgery; (7) cardiac pacemaker; (8) refusal to accept acupuncture and TENS treatment; (9) mental disorder; (10) history of epilepsy and still taking an

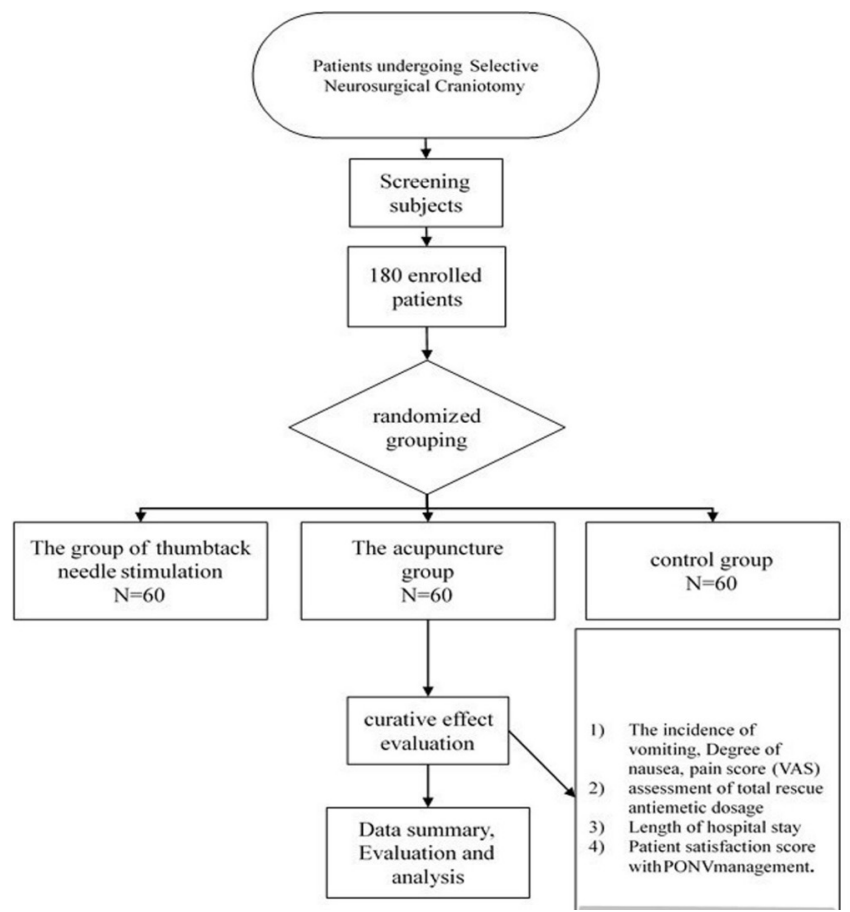

Figure 3 Trial flow chart. PONV, postoperative nausea and vomiting; VAS, visual analogue scale. antiepileptic medicine; (11) unconscious before the surgery; (12) cannot normally communicate; (13) undergoing ventricle or brainstem surgery; (14) cerebral perfusion pressure of greater than $150 \mathrm{~mm} \mathrm{Hg}$ and signs of encephalopathy; (15) poorly controlled diabetes mellitus (fasting plasma glucose greater than $12 \mathrm{mmol} / \mathrm{L}$ ); (16) bleeding disorders (haemophilia or afibrinogenaemia) and (17) serious systemic disease (AIDS or sepsis).

Dropout criteria: Participants who meet any of the following criteria are withdrawn from the study: (1) death; (2) waking up more than 2 hours after surgery; (3) tracheal intubation; (4) persistent coma; (5) cognitive impairment; (6) further surgery or transfer to ICU if necessary for the aggravation of the disease, and so on. Patients who are withdrawn are not replaced.

\section{RANDOMISATION AND BLINDING}

A randomised grouping plan will be designed using the statistical software named Package for Encyclopedia Medical Statistics V.3.1 (PEMS 3.1). Using this plan, 180 patients will be randomly assigned to either an acupuncture, intradermal thumbtack needle or control treatment group. The grouping scheme will be kept hidden in an envelope. The included participants will be randomly assigned to each group according to the distribution scheme in the envelope: 60 patients in each group. This study is a single-blind design, to keep patients unaware of which study group they will be randomly assigned to, and the efficacy evaluator and statistician are separated in this study.

Study investigators, acupuncturists and participants will be aware of the treatment allocation. Outcome assessors and data analysts will be blinded and participants will be asked not to reveal their allocation to assessors. In addition, we will have blinded interpretation of the study results to minimise misleading data interpretation.

\section{INTERVENTIONS}

The determination of the programme and the point of acupuncture is based on previous research and The Name and Location of Acupoints (GB/T 12346-2006). All the practitioners performing the treatment must have an acupuncturist qualification certificate and have performed clinical treatment independently for more than 2 years. The acupuncturists are not replaced during the experiment.

All patients will receive the same anaesthesia methods: general anaesthesia with endotracheal intubation. Blood pressure, heart rate, pulse oximetry and end-tidal $\mathrm{CO}_{2}$ will be routinely monitored. Induction of anaesthesia will be achieved with midazolam $0.05 \mathrm{mg} / \mathrm{kg}$, sufentanil $0.3 \mu \mathrm{g} / \mathrm{kg}$, atracurium $0.15 \mathrm{mg} / \mathrm{kg}$ and propofol $2 \mathrm{mg} /$ $\mathrm{kg}$. When endotracheal intubation and gastrointestinal decompression with either an orogastric or nasogastric tube are undertaken, the anaesthesia will be maintained with $50 \%$ nitrous oxide and 3\% sevoflurane. An attending 


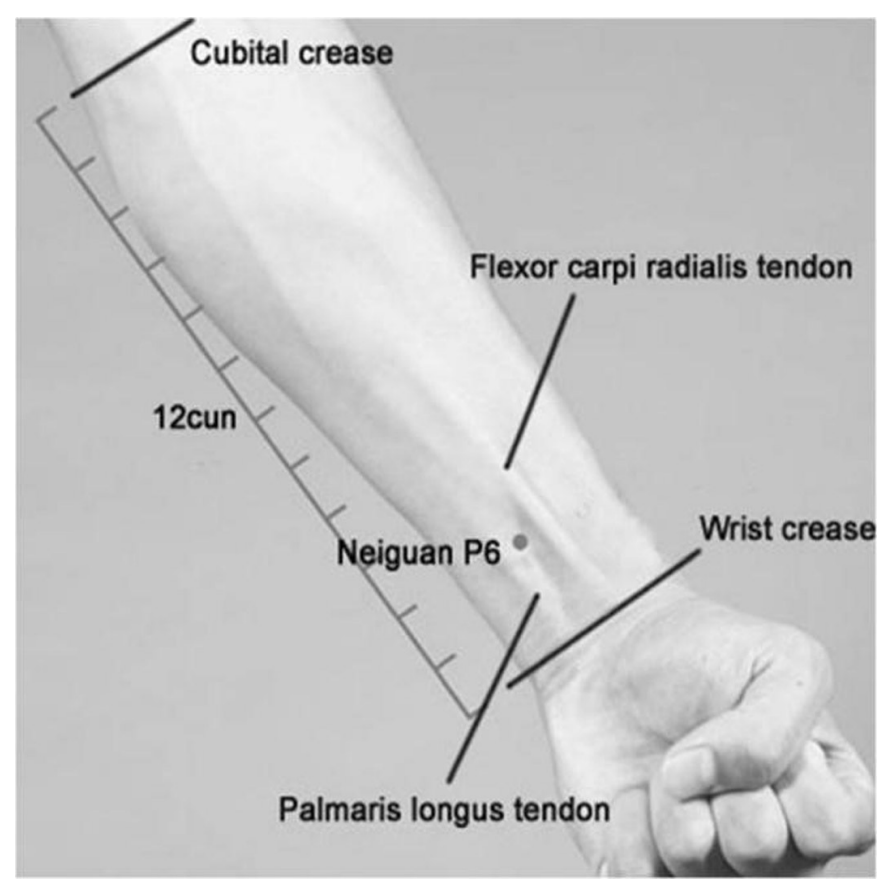

Figure 4 Location of P6 acupoint. P6, pericardium 6.

anesthesiologist will adjust the concentration of sevoflurane according to BIS and the vital signs, if the hypotension happened and the BIS is low, he or she will decrease the sevoflurane. The target range of BIS was 40-60 during surgery. After the operation has commenced, participants will be given sufentanil $0.2 \mu \mathrm{g} / \mathrm{kg}$ and atracurium $0.1 \mathrm{mg}$ / $\mathrm{kg}$ intermittently. Thirty minutes before the end of the operation, the patients will be treated with prophylactic antiemetic drugs: ondansetron hydrochloride tablets $8 \mathrm{mg}$ according to the advice of doctors. After surgery, patients will be continually monitored in the postanaesthesia care unit (PACU) after anaesthesia to continue the ventilator support. The tracheal tube will be removed after the patients awake. The time from the start of anaesthesia induction to the time of removal of the tube will be recorded. Patients who then meet the criteria (Steward Rating Scale $\geq 4$ and the blood gas index of special patients being normal as judged by the anaesthetist) will be sent back to the ward.

For the acupuncture group (acupuncture is added at the base of basic treatment), bilateral Neiguan (P6, located on the line joining Daling and Quze, between the tendons of palmaris longus and flexor carpi radialis, 2 cun above the transverse crease of the wrist) (figure 4) will be selected.

The location of the acupoint refers to national standards of PRC published in 2006 (GB/T 12346-2006) ('The Name and Positioning of Acupoints'). After the patients are transported from the PACU to the common ward, the treatment will commence. After skin disinfection with a disposable disinfecting cotton swab, sterile and disposable stainless steel needles $(0.25 \times 25 \mathrm{~mm}$, Suzhou Hwato-med, Jiangsu, China) will be quickly and perpendicularly inserted into the skin at P6 acupoints bilaterally to a depth of $20 \mathrm{~mm}$. The duration of reinforcing-reducing manipulation of twirling and rotating needle should be used for $1 \mathrm{~min}$ to achieve de qi (a composite of sensations including soreness, numbness, distention, heaviness and other sensations), which is recognised to be an essential component for acupuncture efficacy. The needles will be kept in place for $30 \mathrm{~min}$ and manipulated manually every 10 min to maintain the de qi. When the treatment is over, all needles will be carefully removed. The therapy includes two treatments, and the acupuncture will be administered immediately after and 24 hours after surgery.

For the group of thumbtack needle stimulation (press needle is added at the base of conventional therapy), the bilateral $\mathrm{P} 6$ acupoint will be selected. The location of the acupoint refers to national standards of PRC published in 2006 (GB/T 12346-2006) ('The Name and Positioning of Acupoints'). Treatment will commence after the patients are transported from the PACU to the common ward. After skin cleansing with a $75 \%$ alcohol swab, Japanese Seirin Pyonex $(0.2 \times 1.5 \mathrm{~mm})$ will be quickly inserted into the skin and embedded at $\mathrm{P} 6$ acupoints bilaterally. Patients and their families will be asked to press the needlepoint when the patient feels nausea, experiences vomiting, bloating, pain and other discomforts. The needle will be replaced after 24 hours. The therapy is given two times as the thumbtack needle stimulation will be given immediately after and 24 hours following surgery.

For the control treatment group of rapid rehabilitation surgery, the benefits, specific measures, prevention of complications, time and cost of hospitalisation will be explained to patients and their families in detail using multimedia presentations and pamphlets prior to the operation. Preoperative measures also include fasting for 6 hours, fasting water for 2 hours, and infusing water and carbohydrates 2 hours before surgery. Premedication will not be administered. Fluid volume management and temperature intervention, drainage system, urethral catheter and gastric tube will not be applied during the operation. The postoperative measures include multimode control of postoperative pain, early ambulation, early extubation (when tubes are applied), ADR monitoring and prognosis monitoring.

\section{OUTCOME MEASURES \\ Main outcome}

In this study, patients are monitored for 48 hours postoperatively. The observers will record any episodes of vomiting (criteria based on vomiting action or vomitus in the mouth) $0-2,2-6,6-12,12-24$ and $24-48$ hours following surgery. The incidence of PONV within 48 hours after craniotomy across the three groups is the main criteria to be measured.

\section{Secondary outcome}

The observers will evaluate the patients' degree of nausea using the WHO's PONV fourth class rating scale: (0) no nausea at all; (1) mild nausea or abdominal discomfort 
without vomiting; (2) evident nausea without vomitus and (3) extreme nausea and vomitus containing gastric juice, which is uncontrolled by medicine. The pain score adopts a standard visual analogue scale during $0-2,2-6,6-12$, 12-24 and 24-48 hours after craniotomy under general anaesthesia. The assessment of total rescue antiemetic dosage $0-48$ hours after craniotomy under general anaesthesia will be recorded. The length of stay in the hospital and patient satisfaction score with PONV management will also be recorded.

\section{Adverse event reporting}

Adverse events (AEs) will be recorded via voluntary reports by neurosurgeons, physical examination, laboratory examination or other methods. All AEs must be recorded on the case report form's (CRF's) AEs page and the following information about AEs should be provided: (1) severity degree (mild, moderate or severe); (2) the relationship with research therapy (definitely related, probably related, possibly related, probably irrelevant or definitely irrelevant); (3) duration (the start and end dates or if the AE still exists at the last check); (4) serious $\mathrm{AE}$ (SAE) and (5) important medical events (if potentially harmful to patients, medical or surgical intervention may be requested).

To ensure the safety of each patient, any SAEs that occur from the time the patient gives consent up to 30 days after completion of the study, whether or not associated with the treatment of this research, must be reported to the project director within 24 hours. SAEs that occur 30 days after the cessation of the study do not need to be reported unless the researchers deem it relevant to the treatment. The recurrence, complication or progression of previously reported SAEs must also be reported as first reported follow-up information, as soon as the first SAE occurs. The researchers must report the events within 24 hours once receiving the follow-up information. If a SAE is considered to be completely unrelated to the previously reported one, it should be reported as a new event.

\section{Sample size calculation and statistical analysis}

A German prospective observational study published in 2011 demonstrated an overall incidence of PONV in 47\% of patients after craniotomy under general anaesthesia. The sample size is determined by using PEMS 3.1 with $\alpha=0.05$ (two-sided) and $\beta=0.1$ (90\% power). The formula for calculation is as follows:

$$
\mathrm{n}=2 \lambda /\left(2 \sin ^{-i} \sqrt{P_{\max }}-2 \sin ^{-1} \sqrt{P_{\min }}\right)^{2}
$$

To demonstrate a $30 \%$ absolute reduction in the incidence of PONV, the sample size will be 49 patients for each group. Considering the potential for loss and attrition (20\%), 60 patients per group, or a total of 180 patients, should be reasonable. If a patient cannot complete the study due to death, reoperation, unconsciousness or cognitive impairment, that patient cannot be considered successfully treated and will be recorded as an episode of PONV for statistical purposes.
All data will be analysed by a blinded statistician using PEMS 3.1 at a separate location from the WCHSU. The intention-to-treat principle will be used in the data analysis. Baseline data will be collected and compared first. Different statistics are presented differently, for example, continuous data are presented as mean $( \pm \mathrm{SD})$. $\chi^{2}$ test is used to compare the incidence of postoperative vomiting, complete response rate, sex difference and other nominal data. Conversely, the Kruskal-Wallis test is used to compare the nausea score, satisfaction score and antiemetic dosage. Additionally, the Nemenyi test and Scheffé's method are used for multiple comparisons between groups. A p value $<0.05$ is considered statistically significant.

\section{Patient and public involvement}

The patients and the public were not involved in the planning and design of this study.

The present trial was developed by acupuncturists based on previous clinical experience and the literature. Patients were not involved in the design of the study. The outcomes were commonly used assessments of PONV in clinical practice. The cost of interventions and outcome measurements were mostly covered by the study funding, so it was not thought to be a significant burden and were in line with patient preferences. The results will be disseminated to study participants via the website of our hospital.

\section{DISCUSSION}

Our previous studies have shown the effectiveness and safety of acupuncture in the treatment of nausea and vomiting following craniotomy. In the clinic, however, we have found that, as the time of PONV is not fixed, patients are eager to receive treatment when they experience nausea and vomiting. Often, however, acupuncture cannot be administered as there is no qualified practitioner available. To improve the availability of treatment, acupuncturists need to formulate new protocols. Seaband and TENS are effective treatments for PONV. They are easy to administer and non-invasive, though our previous studies have found that their efficacy is inferior to needle therapy. The intradermal thumbtack needle has a piercing effect and can be in place for 24 hours. When the patient is nauseous, pressing the acupuncture point can achieve de qi sensation. We, therefore, designed this randomised controlled study to demonstrate that intradermal thumbtack needle buried Neiguan point therapy can reduce nausea and vomiting after craniotomy. It is conducive to clinical use and improved patient satisfaction.

\section{Ethics and dissemination}

The CRFs will be stored in a locked cabinet at the hospitals and accessible by the research team only. On completion of the trial and data checking, the CRFs will be transferred to be securely archived at Sichuan University for 5 years before being destroyed. The trial database will 
be anonymised, password-protected and securely held. Patient identifiable data are shared only within the clinical team on a need-to-know basis to provide clinical care and ensure appropriate follow-up. The aggregated research findings will be presented at national and international scientific conferences and be submitted for publication in peer-reviewed journals.

Contributors $\mathrm{J}-\mathrm{qL}$ devised the study question and design. $\mathrm{YY}$ and $\mathrm{YL}$ developed the idea into the full protocol and wrote the article draft. T-hX reviewed the protocol. L-qJ calculated the sample size and specified the statistical strategy. CW is in charge of conducting all the procedures. All the authors read and approved the final manuscript.

Funding This research is supported by the Sichuan Provincial Administration of Traditional Chinese Medicine Research Fund Support (2018QN037) and the National College Students' Innovation and Entrepreneurship Training Program (C2018102074)

Disclaimer The funding bodies do not play roles in study design, data collection, analysis, interpretation of results and the manuscript.

Competing interests None declared.

Patient consent for publication Not required.

Ethics approval Ethical approval has been granted by the West China Hospital of Sichuan University Clinical Trials and Biomedical Ethics Committee in July 2018. The approval number is 2018 (231). The trial protocol strictly follows the principles of the Declaration of Helsinki (version Seoul, 2008).

Provenance and peer review Not commissioned; externally peer reviewed.

Open access This is an open access article distributed in accordance with the Creative Commons Attribution Non Commercial (CC BY-NC 4.0) license, which permits others to distribute, remix, adapt, build upon this work non-commercially, and license their derivative works on different terms, provided the original work is properly cited, appropriate credit is given, any changes made indicated, and the use is non-commercial. See: http://creativecommons.org/licenses/by-nc/4.0/.

ORCID iD

Chengwei Wang http://orcid.org/0000-0002-7887-0781

\section{REFERENCES}

1 Frey UH, Funk M, Löhlein C, et al. Effect of p6 acustimulation on post-operative nausea and vomiting in patients undergoing a laparoscopic cholecystectomy. Acta Anaesthesiol Scand 2009;53:1341-7.

2 Golembiewski J, Chernin E, Chopra T. Prevention and treatment of postoperative nausea and vomiting. Am J Health Syst Pharm 2005;62:1247-60.

3 Post-anaesthesia Care Unit. Causes of postoperative complications of 9136 patients in the Post-anaesthesia care unit. J Chin Med Univ 2011;40:349-51.

4 Sato K, Sai S, Adachi T. Is microvascular decompression surgery a high risk for postoperative nausea and vomiting in patients undergoing craniotomy? J Anesth 2013;27:725-30.

5 Rahimi SY, Alleyne CH, Vernier E, et al. Postoperative pain management with tramadol after craniotomy: evaluation and cost analysis. J Neurosurg 2010;112:268-72.
6 Guang-Qiang C, Qian W, Yi Z. Preventive Medication for Postoperative Vomiting in Patients Undergoing Neurosurgery Craniotomy:A Retrospect Study. Evaluation and Analysis of Drug-Use in Hospitals of China 2009;9:375-6.

7 Misra S, Parthasarathi G, Vilanilam GC. The effect of gabapentin premedication on postoperative nausea, vomiting, and pain in patients on preoperative dexamethasone undergoing craniotomy for intracranial tumors. J Neurosurg Anesthesiol 2013;25:386-91.

8 Ryu J-H, Lee J-E, Lim Y-J, et al. A prospective, randomized, doubleblind, and multicenter trial of prophylactic effects of ramosetronon postoperative nausea and vomiting (PONV) after craniotomy: comparison with ondansetron. BMC Anesthesiol 2014;14:63.

9 Fabling JM, Gan TJ, El-Moalem HE, et al. A randomized, doubleblind comparison of ondansetron versus placebo for prevention of nausea and vomiting after infratentorial craniotomy. J Neurosurg Anesthesiol 2002;14:102-7.

10 Habib AS, Gan TJ. Evidence-Based management of postoperative nausea and vomiting: a review. Can J Anesth/J Can Anesth 2004;51:326-41.

11 Kovac AL. Prevention and treatment of postoperative nausea and vomiting. Drugs 2000;59:213-43.

12 Alizadeh R, Esmaeili S, Shoar S, et al. Acupuncture in preventing postoperative nausea and vomiting: efficacy of two acupuncture points versus a single one. J Acupunct Meridian Stud 2014;7:71-5

13 Ling J, Bailiang Y, Jinfu T. Neiguan Massaged to prevent AChE and nausea and vomit after laparoscopic Cholecystectomy-A randomized controlled clinical trial. J Zhejiang Univ Tradit Chin Med 2010;34:745-6.

14 Nilsson I, Karlsson A, Lindgren L, et al. The efficacy of p6 acupressure with sea-band in reducing postoperative nausea and vomiting in patients undergoing craniotomy: a randomized, double-blinded, placebo-controlled study. J Neurosurg Anesthesiol 2015;27:42-50.

15 Liu X, Li S, Wang B, et al. Intraoperative and postoperative anaesthetic and analgesic effect of multipoint transcutaneous electrical acupuncture stimulation combined with sufentanil anaesthesia in patients undergoing supratentorial craniotomy. Acupunct Med 2015;33:270-6.

16 An L-X, Chen X, Ren X-J, et al. Electro-Acupuncture decreases postoperative pain and improves recovery in patients undergoing a supratentorial craniotomy. Am J Chin Med 2014;42:1099-109.

17 Asmussen S, Maybauer DM, Chen JD, et al. Effects of acupuncture in anesthesia for craniotomy. $J$ Neurosurg Anesthesiol 2017;29:219-27.

18 Gan TJ, Diemunsch P, Habib AS, et al. Consensus guidelines for the management of postoperative nausea and vomiting. Anesth Analg 2014;118:85-113.

19 Cheong KB, Zhang J-ping, Huang Y, et al. The Effectiveness of Acupuncture in Prevention and Treatment of Postoperative Nausea and Vomiting - A Systematic Review and Meta-Analysis. PLoS One 2013;8:e82474

20 Lu Z, Dong H, Wang Q, et al. Perioperative acupuncture modulation: more than anaesthesia. Br J Anaesth 2015;115:183-93.

21 Zhang JQ, Xue FS, Meng FM, et al. Assessing the anaesthetic and analgesic effects of electroacupuncture in patients undergoing craniotomy. Acupunct Med 2016;34:69-70.

22 Jian-Qin L, Hui P, Ning L I. Acupuncture in the treatment of postoperative nausea and vomiting: a report of one case and review of related literature. West Chin Med J 2013;9:1726-30.

23 Lü JQ, Feng R-Z, Pan H, et al. [A randomized controlled clinical trial for acupuncture stimulation of Neiguan (PC 6) to prevent postoperative nausea and vomiting]. Zhen Ci Yan Jiu 2013;38:245.

24 Lv J-qin, Feng R-zhi, Li N. P6 acupoint stimulation for prevention of postoperative nausea and vomiting in patients undergoing craniotomy: study protocol for a randomized controlled trial. Trials 2013;14:153. 